\title{
Patterning of nanodot-arrays using EUV achromatic Talbot lithography at the Swiss Light Source and Shanghai Synchrotron Radiation Facility
}

\author{
$\underline{\text { Daniel Fan*a }}{ }^{\mathrm{a}}$, Elizabeth Buitrago $*^{\mathrm{a}}$, ShuminYang*b ${ }^{\mathrm{b}}$, Waiz Karim ${ }^{\mathrm{ac}}$, Yanqing Wu ${ }^{\mathrm{b}}$, \\ Renzhong Tai ${ }^{\mathrm{b}}$, Yasin Ekinci ${ }^{\mathrm{a}}$ \\ ${ }^{a}$ Laboratory for Micro- and Nanotechnology, Paul Scherrer Institute, 5232 Villigen PSI, Switzerland \\ ${ }^{\mathrm{b}}$ Shanghai Institute of Applied Physics, Shanghai Synchrotron Radiation Facility, 239 Zhangheng Road, \\ Shanghai, China \\ ${ }^{\mathrm{c}}$ Institute for Chemical and Bioengineering, ETH Zurich, Switzerland \\ *main authors, corresponding author: daniel.fan@psi.ch
}

Keywords: EUV, interference lithography, high resolution, nano-lithography, achromatic Talbot lithography

\begin{abstract}
Achromatic Talbot lithography (ATL) at extreme ultraviolet (EUV) wavelengths has been used to produce one or two-dimensional periodic patterns over large areas. In this work, an ATL transmission mask was used to perform EUV exposures at $13.5 \mathrm{~nm}$ and $8.8 \mathrm{~nm}$ illumination wavelengths at two different synchrotron facilities, to study the broadband nature of the method and the used mask as well as to investigate the influence of illumination parameters and experimental arrangements. The experiments were performed at the Swiss Light Source (SLS), PSI, Switzerland, and at the Shanghai Synchrotron Radiation Facility (SSRF), P. R. China. Achromatic Talbot lithography was proven to be a simple and robust interference lithography scheme for
\end{abstract}


producing large area and high resolution patterns suitable for different wavelengths and for a variety of EUV sources and setups.

\section{INTRODUCTION}

There has been an enormous amount of interest in the nanostructures of various materials and particularly towards potential applications for future electronic devices [1]. Extreme ultraviolet (EUV) lithography at the $13.5 \mathrm{~nm}$ wavelength is the best industry candidate for producing next generation electronic devices [2]. Nonetheless, before EUV lithography is introduced into high volume manufacturing many new EUV resists require development and evaluation with EUV light [3]. EUV interference lithography is not only a useful method for research and development of EUV materials and technologies, but it also enables the fabrication of large area high resolution periodic nanostructures $[4,5]$. Achromatic Talbot lithography (ATL), also known as achromatic spatial frequency multiplication (ASFM), is a very robust, highly efficient, and simple technique to produce highly dense, high-resolution periodic nanostructures down to $15 \mathrm{~nm}$ feature size $[6,7]$. This method is suitable for broadband EUV sources, i.e. the majority of EUV sources, and for low intensity or brightness sources, since the aerial image is generated using the interference from all transmitted diffraction orders, allowing large area patterning with high throughput via step-and-repeat exposures [7].

In this study, ATL masks have been fabricated at the Laboratory of Micro and 
Nanotechnology (LMN) at the Paul Scherrer Institute (PSI) in Switzerland. The masks are transmission masks of nickel absorbers on silicon nitride membranes. The highly efficient ATL masks were then first tested at the X-ray interference lithography (XIL) beamline of the Swiss Light Source (SLS) in PSI and subsequently at the IL beamline of the Shanghai Synchrotron Radiation Facility (SSRF), P. R. China, to elucidate and compare the performance of the two beamlines for high-resolution patterning.

\section{THEORY AND CALCULATIONS}

Talbot first noticed that periodic patterns were produced at fixed distances from a diffraction grating upon which monochromatic coherent light was incident [8]. These patterns are self-images of the diffraction grating, produced at repeating Talbot distances at every integer multiple of $z_{T}$ (Eq. 1) from the mask [9]:

$$
z_{T}=\frac{2 p^{2}}{\lambda}
$$

where $p$ is the grating period and $\lambda$ is the illumination wavelength (Fig. 1). 


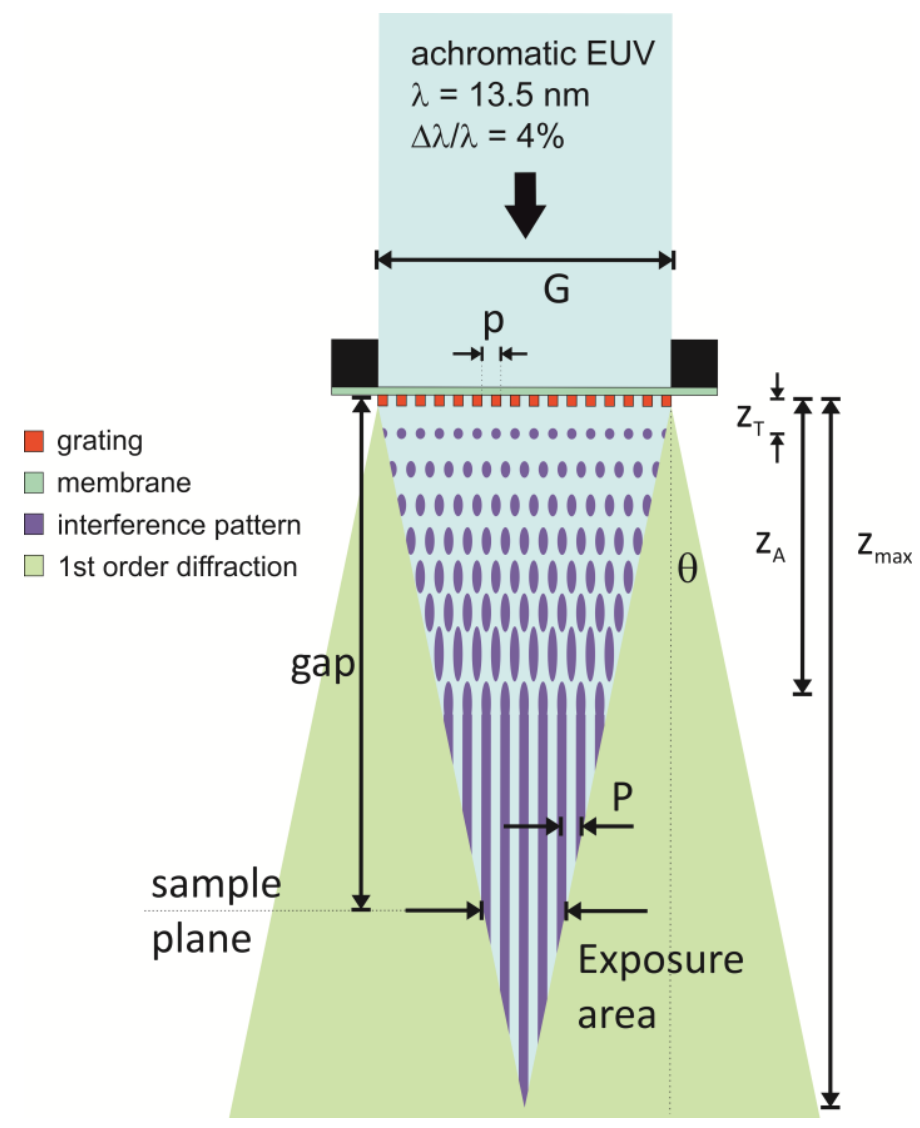

Figure 1 (single column). Scheme of achromatic Talbot lithography. Self-images of the diffraction grating are produced at the Talbot distance $z_{T}$ from the grating, after which they smear out and eventually become stationary images at the achromatic Talbot distance $z_{A}$.

When the incident light has a spectral bandwidth of $\Delta \lambda$, the Talbot images due to different incident wavelengths overlap at a certain distance. This occurs when the Talbot distance of one image due to the minimum incident wavelength equals the Talbot distance of a subsequent image due to the maximum incident wavelength (Eq. 2):

$$
\frac{2 n p^{2}}{\lambda-\Delta \lambda / 2}=\frac{2(n+1) p^{2}}{\lambda+\Delta \lambda / 2}=z_{A}
$$

where $n$ is an integer for every subsequent self-image starting from the grating plane. Solving for $n$, substituting back into Eq. 2, and assuming $\lambda / \Delta \lambda$ is much larger 
than $1 / 2$ gives the achromatic Talbot distance $z_{A}$ (Eq. 3). Beyond this distance, self-images of the grating smear and overlap in the $z$-direction and the aerial image becomes $z$-invariant beyond $z_{A}[10]$ :

$$
z_{A}=\frac{2 p^{2}}{\Delta \lambda}
$$

This is the minimum distance that is needed to obtain a $z$-stationary aerial image. As seen in Eq. 3, the bandwidth of the source turns into an advantage by providing an aerial image of very large depth-of-focus. As the self-images are due to the interference from all transmitted diffraction orders, all of the transmitted intensity contributes to the aerial image, and therefore exposures are highly efficient in comparison for example to multiple-beam interference lithography. Furthermore, the whole aerial image can be patterned, giving large-area patterning capabilities in comparison to multiple-beam interference lithography where only the interference patterns due to first or second order diffraction is recorded while the exposed areas due to zeroth order diffraction are not patterned. For a line/space diffraction grating, the resulting line/space pattern has a pitch that is $1 / 2$ of the mask grating period, while for a dot/hole diffraction grating, the resulting dot/hole array pattern has a pitch that is $1 / \sqrt{ } 2$ of the mask grating period rotated by $45^{\circ}$ [10]. We note that for both dot and hole array diffraction gratings, a dot array is obtained using negative resist.

From the definition of $z_{A}$ presented above, it is clear that the grating periodicity and the bandwidth of illumination influences the achromatic Talbot distance. In addition, 
it should be noted that the aerial image in ATL is a result of interference of diffraction orders from the same grating. As the diffraction orders diverge from each other when moving away from the grating, the overlapping area of diffraction orders and thereby the area of the interference decreases. The diffraction angle $\theta$ of a grating with period $p$ and with illumination wavelength $\lambda$ is calculated by the Bragg equation:

$$
\frac{\lambda}{p}=\sin \theta
$$

Given the diffraction angle, the field size can be calculated from the gap between mask and sample plane, or vice versa:

$$
\tan \theta=\frac{G-x}{2 z}
$$

where $x$ is the side length of the patterned area, $G$ is the side length of the grating, and $z$ is the gap distance between mask and sample plane. As the distance of the sample plane from the mask increases, the patterned area $x^{2}$ approaches zero at the maximum distance $z_{\max }$. For small diffraction angles, $z_{\max }$ is given by combining Eq. 4 and Eq. 5 to give:

$$
z_{\max }=G \times \frac{p}{2 \lambda}
$$

For ATL, the distance between the mask and the sample plane should therefore be larger than the achromatic Talbot distance but smaller than the maximum distance (Figure 1). This distance should be kept short in order to maximize the size of the patterned area. For a diffraction grating with period $150 \mathrm{~nm}$ and a size of $100 \times 100$ 
$\mu \mathrm{m}^{2}$, the calculations for the relevant ATL parameters are given in Table 1 .

Table 1: Calculations for EUV exposure at PSI and SSRF using $100 \times 100 \mu \mathrm{m}^{2}$ ATL mask with $150 \mathrm{~nm}$ period (i.e. $G=100 \mu \mathrm{m}, p=150 \mathrm{~nm}$ ).

\begin{tabular}{|ccccc|}
\hline Parameter & Equation & PSI & SSRF $\lambda_{1}$ & SSRF $\lambda_{2}$ \\
\hline \hline Illumination wavelength & $\lambda$ & $13.5 \mathrm{~nm}$ & $13.5 \mathrm{~nm}$ & $8.8 \mathrm{~nm}$ \\
\hline Image period $\left(45^{\circ}\right)$ & $P=\frac{p}{\sqrt{2}}$ & $106 \mathrm{~nm}$ & $106 \mathrm{~nm}$ & $106 \mathrm{~nm}$ \\
\hline Spectral bandwidth & $\Delta \lambda / \lambda$ & $4 \%$ & $3 \%$ & $3 \%$ \\
\hline Monochromatic Talbot distance & $Z_{T}=\frac{2 p^{2}}{\lambda}$ & $3.3 \mu \mathrm{m}$ & $3.3 \mu \mathrm{m}$ & $5.1 \mu \mathrm{m}$ \\
\hline Achromatic Talbot distance & $Z_{A}=\frac{2 p^{2}}{\Delta \lambda}$ & $83.3 \mu \mathrm{m}$ & $111 \mu \mathrm{m}$ & $170 \mu \mathrm{m}$ \\
\hline Maximum distance & $Z_{\text {max }}=G \times \frac{p}{2 \lambda}$ & $556 \mu \mathrm{m}$ & $556 \mu \mathrm{m}$ & $852 \mu \mathrm{m}$ \\
\hline
\end{tabular}

\section{MATERIALS AND METHODS}

Achromatic Talbot lithography was performed at the XIL-II beamline, Swiss Light Source, Paul Scherrer Institute, Switzerland, and the IL beamline, Shanghai Synchrotron Radiation Facility, P. R. China, using the same ATL mask.In general, EUV radiation produced from an undulator source is filtered harmonically and spatially by sets of mirrors and pinholes. The XIL-beamline at the SLS has an undulator source filtered by a pinhole to produce spatially coherent light of tunable wavelength/energy $(70-500 \mathrm{eV})$ and a $4 \%$ bandwidth $(\Delta \lambda / \lambda)$. The details are described elsewhere [11]. The SSRF is a third generation $3.5 \mathrm{GeV}$ synchrotron source, the first of its kind in China. The IL beamline at the SSRF is a branch of the soft X-ray spectromicroscopy beamline (STXM) [12], and uses an elliptically polarized undulator (EPU) source. By using the same EPU the energy range can be tuned from $85 \mathrm{eV}$ to $150 \mathrm{eV}$. The beamline layout has been described in detail elsewhere [13]. A 
four-knife slit placed about nine meters in front of the mask acts as an intermediate light source with high spatial coherence. For the ATL exposure, the beamline was set to provide illumination with a central wavelength of $13.5 \mathrm{~nm}$ and $8.8 \mathrm{~nm}$. An exact comparison of the two beamlines is shown in Table 2 .

Table 2: EUV-IL beamlines at PSI and SSRF.

\begin{tabular}{|c|c|c|}
\hline & PSI & SSRF \\
\hline Source & $\begin{array}{l}\text { Linear polarized } \\
\text { undulator }\end{array}$ & $\begin{array}{l}\text { Elliptically polarized } \\
\text { undulator }\end{array}$ \\
\hline Photon energy & 70 to $500 \mathrm{eV}$ & 85 to $150 \mathrm{eV}$ \\
\hline Spot size & $2 \times 3 \mathrm{~mm}^{2}$ & $3 \times 3 \mathrm{~mm}^{2}$ \\
\hline Photon flux at $92 \mathrm{eV}$ & $\begin{array}{c}3 \times 10^{15} \mathrm{~s}^{-1} \mathrm{~cm}^{-2} / 4 \% \mathrm{BW} \\
/ 0.3 \mathrm{~A}\end{array}$ & $\begin{array}{l}3.6 \times 10^{15} \mathrm{~s}^{-1} \mathrm{~cm}^{-2} / \\
2.6 \% \mathrm{BW} / 0.3 \mathrm{~A}\end{array}$ \\
\hline $\begin{array}{l}\text { Gap (distance between mask and } \\
\text { wafer) positioning }\end{array}$ & $\mu \mathrm{m}$ accuracy & $\mathrm{mm}$ accuracy \\
\hline $\begin{array}{l}\text { Effective source size and source to } \\
\text { mask distance }\end{array}$ & $\begin{array}{c}70 \mu \mathrm{m} \text { diameter pinhole } \\
\text { @ } 12 \mathrm{~m}\end{array}$ & $\begin{array}{c}45 \times 45 \mu \mathrm{m}^{2} \text { four-knife } \\
\text { slit @ } 9 \mathrm{~m}\end{array}$ \\
\hline
\end{tabular}

From the geometry described above, the source extension on the pattern can be calculated to be $\sim 3 \mathrm{~nm}$ at the maximum possible mask to sample gap of $550 \mu \mathrm{m}$ for $13.5 \mathrm{~nm}$ incidence wavelength and $\sim 5 \mathrm{~nm}$ at the maximum mask to sample gap of 850 $\mu \mathrm{m}$ for $8.8 \mathrm{~nm}$ incidence wavelength, as per equation 5 ,

$$
\frac{S_{\text {ext }}}{Z}=\frac{S}{L}
$$

where $s$ is the size of the source, $s_{\text {ext }}$ is the size of the source extension on sample wafer, $L$ is the distance from source to mask, and $z$ is the distance from mask to sample wafer. 
Therefore, for beamlines with a similar arrangement as the SLS and SSRF, where the effective source size is much smaller than the source to mask distance, the source extension, i.e. spatial coherence onto the sample introduces a blur of approximately a few nm. For this ATL mask producing a period of $106 \mathrm{~nm}$, a few $\mathrm{nm}$ of blur is relatively small and would not affect the resolution. The effect of source extension becomes a limiting factor for plasma sources, where the source must be placed closer to the mask due to efficiency problems.

The mask fabrication process is shown in Fig. 2 and described elsewhere [7]. Briefly, layered thin films of chromium $(3 \mathrm{~nm})$, gold $(5 \mathrm{~nm})$, and chromium $(3 \mathrm{~nm})$ are thermally evaporated on a $3 \times 3 \mathrm{~mm}^{2}, 100-\mathrm{nm}$-thick silicon nitride membrane. The chromium layers are deposited to improve adhesion and the gold acts as subsequent electroplating seed layer. Next, hydrogen silsesquioxane (HSQ), a high-resolution negative-tone photoresist (Fox16, Dow Corning, Midland MI, U.S.A.), mixed 1:2 with MIBK solvent (Dow Corning) is applied by spin coating at $3000 \mathrm{rpm}$ for a target thickness of $\sim 250 \mathrm{~nm}$. Using e-beam lithography (EBPG 5000+, Vistec, Jena, Germany) a periodic pillar array, with $150 \mathrm{~nm}$ pitch and $75 \mathrm{~nm}$ pillar diameter, was written at $12000 \mu \mathrm{C} / \mathrm{cm}^{2}$ dose over an area of $100 \times 100 \mu \mathrm{m}^{2}$ and then developed in an $\mathrm{NaOH}$ buffered developer (Microposit 351, Dow) diluted 1:3 with deionised (DI) water for $30 \mathrm{~s}$. Using $\mathrm{Cl}_{2}$-based reactive ion etching for $10 \mathrm{~s}$ removed the chromium layer to expose the gold electroplating seed layer. Approximately $200 \mathrm{~nm}$ of nickel was then electroplated at $\sim 2.5 \mathrm{~mA} / \mathrm{cm}^{2}$ with $67 \%$ duty cycle unipolar pulses for $6 \mathrm{~min}$ 
[14]. Nickel is used because it is an excellent photon-stop material due to its high absorption coefficient at EUV wavelengths and therefore provides high contrast with a relatively smaller thickness compared to other materials such as gold, thereby relaxing the process parameters and causing less stress on the membrane support [15]. It is also insensitive to surface roughness, as the absorption coefficient becomes relatively constant above a certain thickness. The theoretical contrast provided by 200 $\mathrm{nm}$ thick nickel was calculated to be approximately $70 \%$ at $500 \mu \mathrm{m}$ propagation distance using $4 \%$ bandwidth (Fig. 3) [10]. The calculated background was approximately $18 \%$ and the contrast and background is invariant in the z-direction. The exposed HSQ structures were then removed in a buffered oxide etch bath (HF 1:7 with $\mathrm{NH}_{4} \mathrm{~F}$ ) with an etch rate of $1 \mathrm{~nm} / \mathrm{s}$. A short reactive ion etch using argon removed the exposed gold and chromium layers to improve mask efficiency. This left a nickel hole pattern with $150 \mathrm{~nm}$ pitch. The mask was mounted on a stainless steel mask holder using PMMA and heated to $50^{\circ} \mathrm{C}$ for solvent removal.

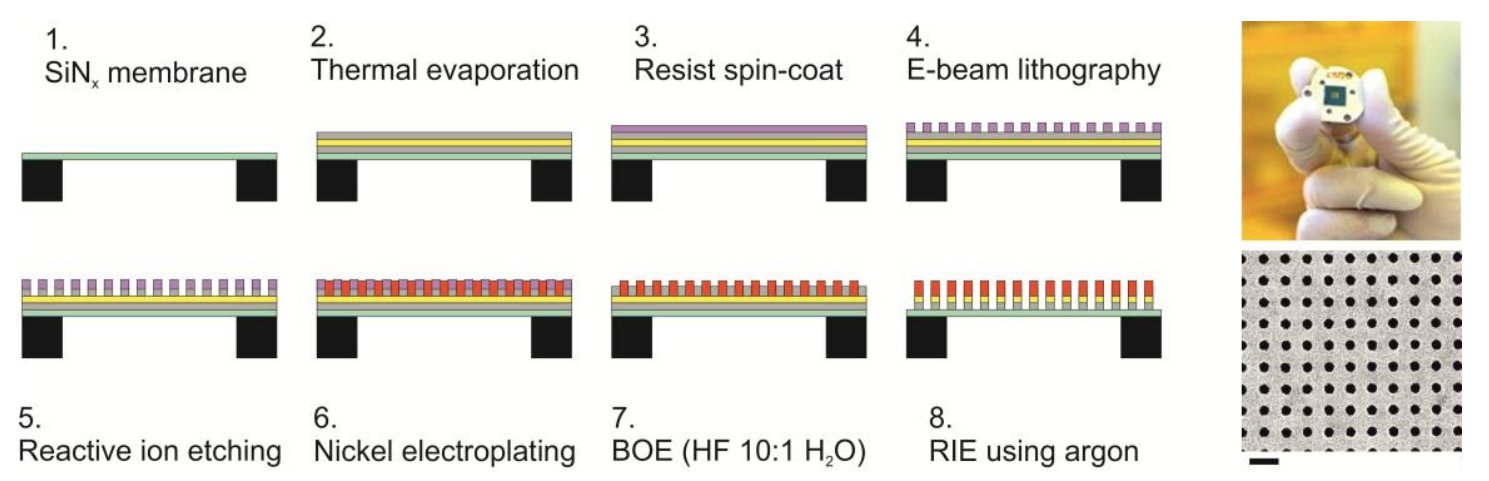

Figure 2 (double column). ATL mask fabrication process. (Top right) ATL mask on stainless steel mask holder. (Bottom right) Scanning electron microscope (SEM) image of nickel ATL mask showing a 2D hole grating with $150 \mathrm{~nm}$ pitch (scale bar $=200 \mathrm{~nm})$. 


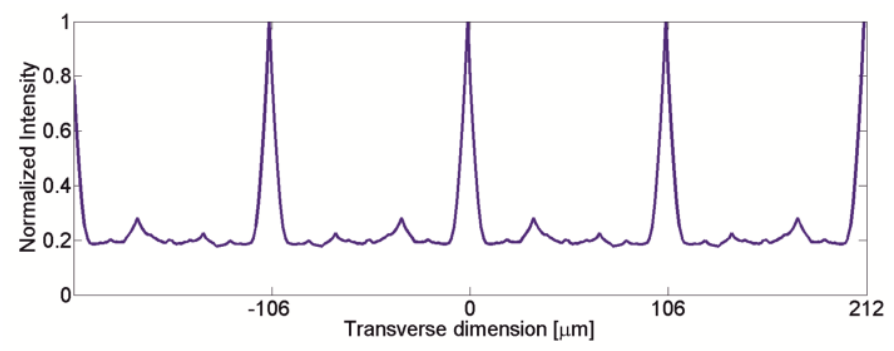

Figure 3 (single column). ATL simulation showing diagonal intensity profile of the aerial image at a propagation distance of $z=500 \mu \mathrm{m}$. The contrast is $70 \%$ with a $18 \%$ background.

\section{RESULTS AND DISCUSSION}

HSQ-coated silicon wafers were used for the exposures at the two beamlines. At the XIL-II beamline at PSI, HSQ (XR1541) was spin-coated at $5000 \mathrm{rpm}$ for $45 \mathrm{~s}$ targeting a thickness of $\sim 35 \mathrm{~nm}$. The gap between ATL mask and sample wafer was set to $275 \mu \mathrm{m}$. The exposure doses ranged from $100 \mathrm{~mJ} / \mathrm{cm}^{2}$ up to $2000 \mathrm{~mJ} / \mathrm{cm}^{2}$ in $100 \mathrm{~mJ} / \mathrm{cm}^{2}$ steps. The measured beam flux was approximately $31 \mathrm{~mW} / \mathrm{cm}^{2}$ giving exposure times from $3 \mathrm{~s}$ to $60 \mathrm{~s}$. The wafers were subsequently developed in $\mathrm{NaOH}$ buffered developer (Microposit 351) diluted 1:3 with DI water for 30 s. SEM inspection of the developed samples show a minimum feature size of $20 \mathrm{~nm}$ over an exposure area of $43 \mu \mathrm{m}$ width (Fig. 4d-f). The resulting feature sizes can be controlled by changing the dose from 600 to $800 \mathrm{~mJ} / \mathrm{cm}^{2}$. Higher doses showed oval dots and astigmatism. The observed field area of $43 \times 43 \mu \mathrm{m}^{2}$ was slightly smaller than expected from calculations given a mask to wafer distance of $275 \mu \mathrm{m}$ and a mask field area of $100 \times 100 \mu \mathrm{m}^{2}$, calculated to be $50 \mu \mathrm{m}$ on wafer. This discrepancy may be due to errors in measurement of the mask thickness and silicon wafer thickness. The gap calculated from the measured field area of $43 \times 43 \mu \mathrm{m}^{2}$ is $318 \mu \mathrm{m}$. 
At the IL branch line at SSRF, HSQ (XR1541-002) was spin-coated on silicon wafers at $4000 \mathrm{rpm}$ for a final thickness of $\sim 60 \mathrm{~nm}$. The wafer was then baked at $180^{\circ} \mathrm{C}$ for 5 min. The exact distance between the mask and the wafer can only be controlled by using a grooved holder. The groove height determines the gap between the mask and the wafer when the exposure is performed in contact mode. To achieve the smallest possible feature size and avoid missing the optimal dose due to the natural vibration of the tool, a $10 \%$ step size in dose was used, starting from $280 \mathrm{~mJ} / \mathrm{cm}^{2}$ up to $1700 \mathrm{~mJ} / \mathrm{cm}^{2}$ in $28 \mathrm{~mJ} / \mathrm{cm}^{2}$ steps. The measured beam flux was approximately 40 $\mathrm{mW} / \mathrm{cm}^{2}$ (exposure time from $7 \mathrm{~s}$ up to $42 \mathrm{~s}$ ). The exposed wafers were developed in TMAH of $25 \%$ concentration for $45 \mathrm{~s}$ and rinsed in DI water. SEM inspection of the developed sample shows dots with feature sizes down to $20 \mathrm{~nm}$ with good uniformity (Fig. 4a). By increasing the exposure dose from 400 to $700 \mathrm{~mJ} / \mathrm{cm}^{2}$ the diameter of the dot array can be increased to $48 \mathrm{~nm}$ (Fig. 4b), in good agreement with simulations [10]. The exposure area was measured to be $32 \mu \mathrm{m}$ (Fig. 4c). 

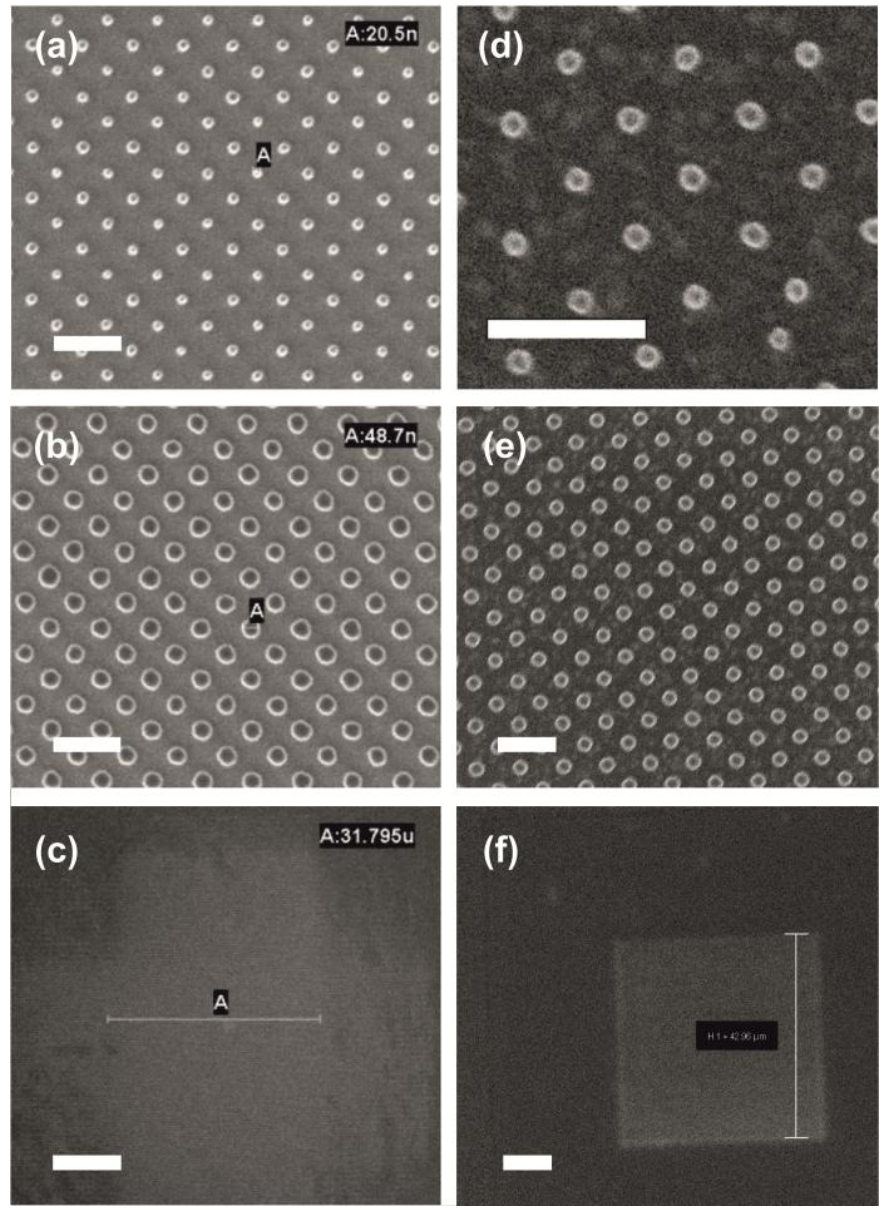

Figure 4 (single column). SEM images of the exposed patterns after development at the SSRF (a-c) and at PSI (d-f). In all cases the dot array has pitch $106 \mathrm{~nm}$. (a) Dose $400 \mathrm{~mJ} / \mathrm{cm}^{2}$, dot size $20 \mathrm{~nm}$. (b) Dose $670 \mathrm{~mJ} / \mathrm{cm}^{2}$, dot size $48 \mathrm{~nm}$. (d) Dose $600 \mathrm{~mJ} / \mathrm{cm}^{2}$, dot size $20 \mathrm{~nm}$. (e) Dose $800 \mathrm{~mJ} / \mathrm{cm}^{2}$, dot size $40 \mathrm{~nm}$. The measured exposure area was (c) $32 \mu \mathrm{m}$ at SSRF and (f) $43 \mu \mathrm{m}$ at PSI. Scale bars for (a-b) and (d-e) equals $200 \mathrm{~nm}$. Scale bars for (c) and (f) equals $10 \mu \mathrm{m}$.

An ATL exposure at a wavelength of $8.8 \mathrm{~nm}$ with a spectral width of $3 \%$ was also tested at the SSRF (Fig. 5) using the same mask and the same wafer gap distance. The shorter wavelength has the advantage that $z_{\max }$ increases even though $z_{A}$ remains the same, thus increasing the depth-of-field. By increasing the exposure dose from 280 $\mathrm{mJ} / \mathrm{cm}^{2}$ in $28 \mathrm{~mJ} / \mathrm{cm}^{2}$ steps, the dot diameter can be increased from $28 \mathrm{~nm}$ to $48 \mathrm{~nm}$ (Fig. 5a-c). The exposure area was measured to be $42 \mu \mathrm{m}$ (Fig. 5d), which is larger than the $32 \mu \mathrm{m}$ measured using $13.5 \mathrm{~nm}$ light. When the exposure wavelength 
becomes shorter, the diffraction angle is smaller and the exposure area then becomes larger. From the measured exposure field sizes, the gap between mask and wafer is calculated to be $380 \mu \mathrm{m}$ for the exposure at $13.5 \mathrm{~nm}$, and $495 \mu \mathrm{m}$ for the exposure at $8.8 \mathrm{~nm}$.

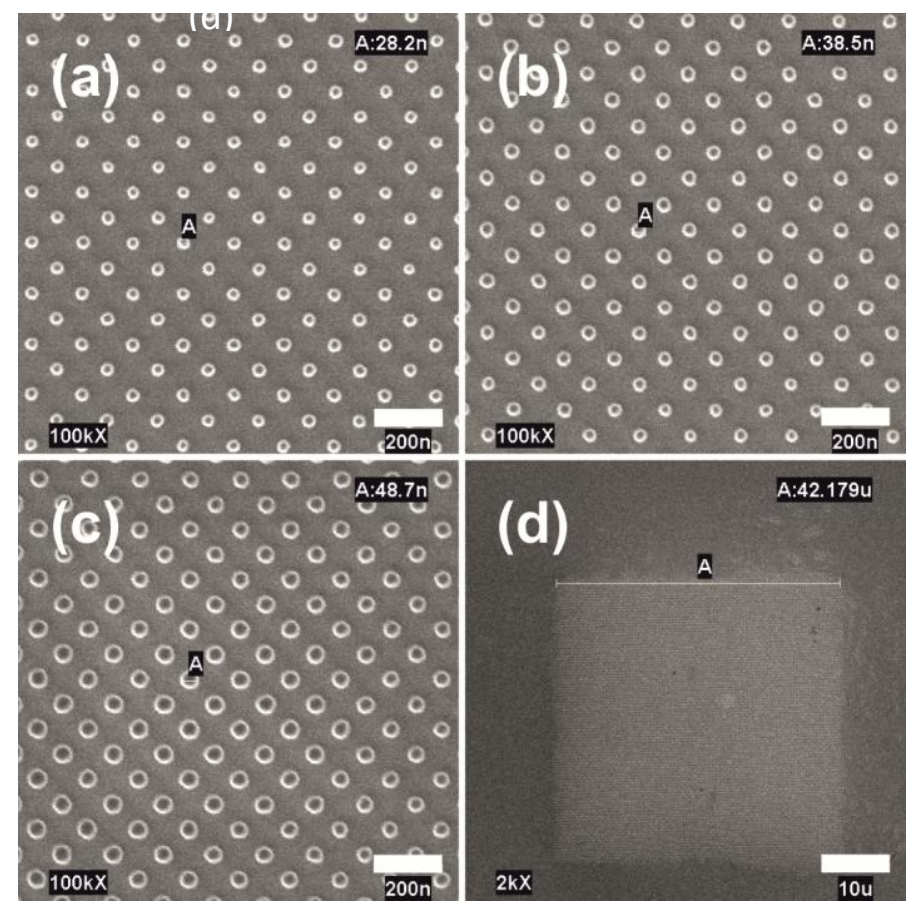

Figure 5 (single column). SEM images of the exposed patterns after development at the SSRF at 8.8 $\mathrm{nm}$ illumination wavelength. In all cases the dot array has pitch $106 \mathrm{~nm}$. (a) Dose $280 \mathrm{~mJ} / \mathrm{cm}^{2}$, dot size $28 \mathrm{~nm}$. (b) Dose $340 \mathrm{~mJ} / \mathrm{cm}^{2}$, dot size $38 \mathrm{~nm}$. (c) Dose $400 \mathrm{~mJ} / \mathrm{cm}^{2}$, dot size $48 \mathrm{~nm}$ and the exposure area is $42 \mu \mathrm{m}$ (d). Scale bars for (a-c) is $200 \mathrm{~nm}$ and (d) is $10 \mu \mathrm{m}$.

Although the distance between the wafer and the mask can be controlled by a mask motor at PSI, the setup at the SSRF does not permit the exact positioning of the mask, making direct comparison of the resulting exposed patterns difficult as different settings had to be used to test the performance of the mask. As can be seen from Figs. 3 and 4, the achievable contrast for the different experiments is similar. An absolute direct comparison of the lithographic performance between the two is not possible 
because of the dissimilarities between the two different IL setups and infrastructure available to each lab the absolute direct comparison of the lithographic performance between the two is not possible. Nevertheless, because the contrast was comparable between the different beamlines and beamline arrangements, this shows that the two beamlines have a similar and comparable performance, and that high resolution periodic patterning was achieved at both synchrotron radiation facilities.

Table 3: Summary of process parameters.

\begin{tabular}{|lcc|}
\hline & PSI (13.5 nm at 4\% bandwidth) & $\begin{array}{c}\text { SSRF (13.5 nm and 8.8 nm at } \\
3 \% \text { bandwidth) }\end{array}$ \\
\hline \hline Photo-resist & HSQ XR1541 & HSQ XR1541-002 \\
\hline Spin-coating & $5000 \mathrm{rpm}$ & $4000 \mathrm{rpm}$ \\
\hline Target thickness & $\sim 35 \mathrm{~nm}$ & $180^{\circ} \mathrm{C}$ \\
\hline PAB & None & 400 to $700 \mathrm{~mJ} / \mathrm{cm}^{2}$ \\
\hline Exposure dose & 600 to $800 \mathrm{~mJ} / \mathrm{cm}^{2}$ & - \\
\hline Mask to sample gap & $275 \mathrm{um}$ & TMAH $25 \% ; 45 \mathrm{~s}$ \\
\hline Developer and time & Microposit $3511: 3 \mathrm{DI}$ water; $30 \mathrm{~s}$ & \\
\hline
\end{tabular}

Effectively, the resolution of the pattern is limited by the resolution of the mask. Writing dense, periodic structures using e-beam lithography is challenging due to the electron proximity effect. Electroplating of the dense structures is also difficult, and for nickel we could achieve $70 \mathrm{~nm}$ feature sizes with good quality. Aspect ratio is also important as HSQ is used as an electroplating mould. $200 \mathrm{~nm}$ of electroplated material is needed, and for feature sizes below $50 \mathrm{~nm}$ requires aspect ratios greater than 4:1. For HSQ, aspect ratios of 3:1 are generally achievable without the need for pattern 
collapse mitigation techniques such as critical point drying. The exposure efficiency could be further increased by process optimization of the mask fabrication. The grating area was kept small as this was a proof-of-concept work for beamline comparison such that the e-beam writing time was kept relatively short. In essence the grating area can be extended to at most the size of the beam spot. The precision of the exposure area delimitation in the context of step-and-repeat exposures is limited by the precision of the sample stage. The minimum period of features patterned using ATL at PSI is $70 \mathrm{~nm}$ with $15 \mathrm{~nm}$ dot size [10], while the theoretical limit approaches $7.5 \mathrm{~nm}$ half-pitch using $10.9 \mathrm{~nm}$ EUV light [16].

\section{CONCLUSIONS}

Achromatic Talbot lithography was performed at PSI and at the SSRF with the same mask. Periodic dot arrays with $20 \mathrm{~nm}$ feature size at $106 \mathrm{~nm}$ pitch were produced using HSQ resist exposed at $13.5 \mathrm{~nm}$ and $8.8 \mathrm{~nm}$ illumination wavelengths. A $3 \%$ bandwidth at SSRF in comparison to $4 \%$ bandwidth at PSI means that the achromatic distance is larger at SSRF which in turn reduces the size of the patterned area and increases the minimum achievable feature size. The scheme is shown to be highly efficient and robust and requires only a broadband EUV source, as well as being equally effective at other EUV wavelengths such as $8.8 \mathrm{~nm}$ and for different illumination parameters. ATL has been proven to be a robust method for use in different types of EUV beamlines, as well as producing high-density, high resolution periodic nanostructures with high throughput. The present study shows that ATL is an excellent tool in the toolbox of EUV lithography and nanofabrication, providing 
high-resolution patterns with different tools and conditions at different wavelengths.

\section{ACKNOWLEDGEMENTS}

Part of this work was performed at the Swiss Light Source (SLS), Paul Scherrer Institute, Switzerland, and at the Shanghai Synchrotron Radiation Facility (SSRF), Shanghai, P. R. China. Financial support from the National Key Basic Research Program of China (2012CB825700) and the Swiss National Science Foundation (200021_143969). Support from Open Research Project of Large Scientific Facility from Chinese Academy of Sciences: Study on Self-assembly Technology and Nanometer Array with Ultra-high Density. D.F. would like to thank Michael Gerspach for assistance with figure illustrations.

\section{REFERENCES}

[1] International Technology Roadmap for Semiconductors 2013 Report [online] 2014, http://www.itrs.net (accessed: 07 October 2015).

[2] G. Tallents, E. Wagenaars, G. Pert, "Optical lithography: lithography at EUV wavelengths," Nat. Photon., vol. 4, pg. 809, 2009.

[3] N. Mojarad, D. Fan, J. Gobrecht, Y. Ekinci, "Broadband interference lithography at extreme ultraviolet and soft x-ray wavelengths," Opt. Lett., vol. 39, no. 8, pp. 2286-2289, April, 2014. 
[4] N. Mojarad, M. Hojeij, L. Wang, J. Gobrecht, Y. Ekinci, "Single-digit-resolution nanopatterning with extreme ultraviolet light for the $2.5 \mathrm{~nm}$ technology node and beyond," Nanoscale, vol. 7, no. 9, pp. 4031-4037, March 2015.

[5] N. Mojarad, J. Gobrecht, Y. Ekinci, "Interference lithography at EUV and soft x-ray wavelengths,” Microelectron. Eng., vol. 143, n. C, pp. 55-63, August 2015.

[6] H. H. Solak, Y. Ekinci, “Achromatic spatial frequency multiplication: a method for production of nanometer-scale periodic structures," J. Vac. Sci. Technol. B, vol. 23, no. 6, pp. 2705-2710, 2005.

[7] W. Karim, S. A. Tschupp, M. Oezaslan, T. J. Schmidt, J. Gobrecht, J. A. van Bokhoven, Y. Ekinci, "High-resolution and large-area nanoparticle arrays using EUV interference lithography," Nanoscale, vol. 7, no. 16, pp. 7386-7393, 2015.

[8] H. F. Talbot, "Facts relating to optical science no. IV," Philos. Mag. 3, vol. 9, no. 56, pp. 401-407, 1836.

[9] Lord Rayleigh, "On copying diffraction-gratings, and on some phenomenon connected therewith," Philos. Mag. 11, pp. 196-205, 1881.

[10]L. Wang, B. Terhalle, M. Hojeij, V. A. Guzenko, Y. Ekinci, "High-resolution nanopatterning by achromatic spatial frequency multiplication with electroplated grating structures," J. Vac. Sci. Technol. B, vol. 30, pg. 031603, 2012.

[11]V. Auzelyte, C. Dais, P. Farquet, D. Grutzmacher, L. J. Heyderman, F. Luo, S. Olliges, C. Padeste, P. K. Sahoo, T. Thomson, A. Turchanin, C. David, H. H. Solak, "Extreme ultraviolet interference lithography at the Paul Scherrer Institut," J Micro Nanolithogr MEMS MOEMS, vol. 8, no. 2, pg. 021204, 2009. 
[12]C. F. Xue, Y. Wang, Z. Guo, Y. Q. Wu, X. J. Zhen, M. Chen, J. H. Chen, S. Xue, Z. Q. Peng, Q. P. Lu, R. Z. Tai, "High-performance soft x-ray spectromicroscopy beamline at SSRF," Rev. Sci. Instrum., vol. 81, no. 10, pg. 103502, 2010.

[13]S. M. Yang, L. S. Wang, J. Zhao, C. F. Xue, H. G. Liu, Z. J. Xu, Y. Q. Wu, R. Z. Tai, "Developments at SSRF in soft x-ray interference lithography," Nucl. Sci. Tech., vol. 26, no. 1, pp. 5-11, 2015.

[14]K. Hili, D. Fan, V. A. Guzenko, Y. Ekinci, "Nickel electroplating for high-resolution nanostructures," Microelectron. Eng., vol. 141, n. C, pp. 122-128, June 2015 .

[15]E. M. Di Fabrizio, F. Romanato, M. Gentili, S. Cabrini, B. Kaulich, J. Susini, "High-efficiency multilevel zone plates for keV x-rays," Nature, vol. 401, no. 6756, pp. 895-898, 1999.

[16] S. Danylyuk, P. Loosen, K. Bergmann, H.-S. Kim, L. Juschkin, "Scalability limits of Talbot lithography with plasma-based extreme ultraviolet sources," J. Micro-Nanolith MEM, vol. 12, no. 3, pp. 033002, 2013. 

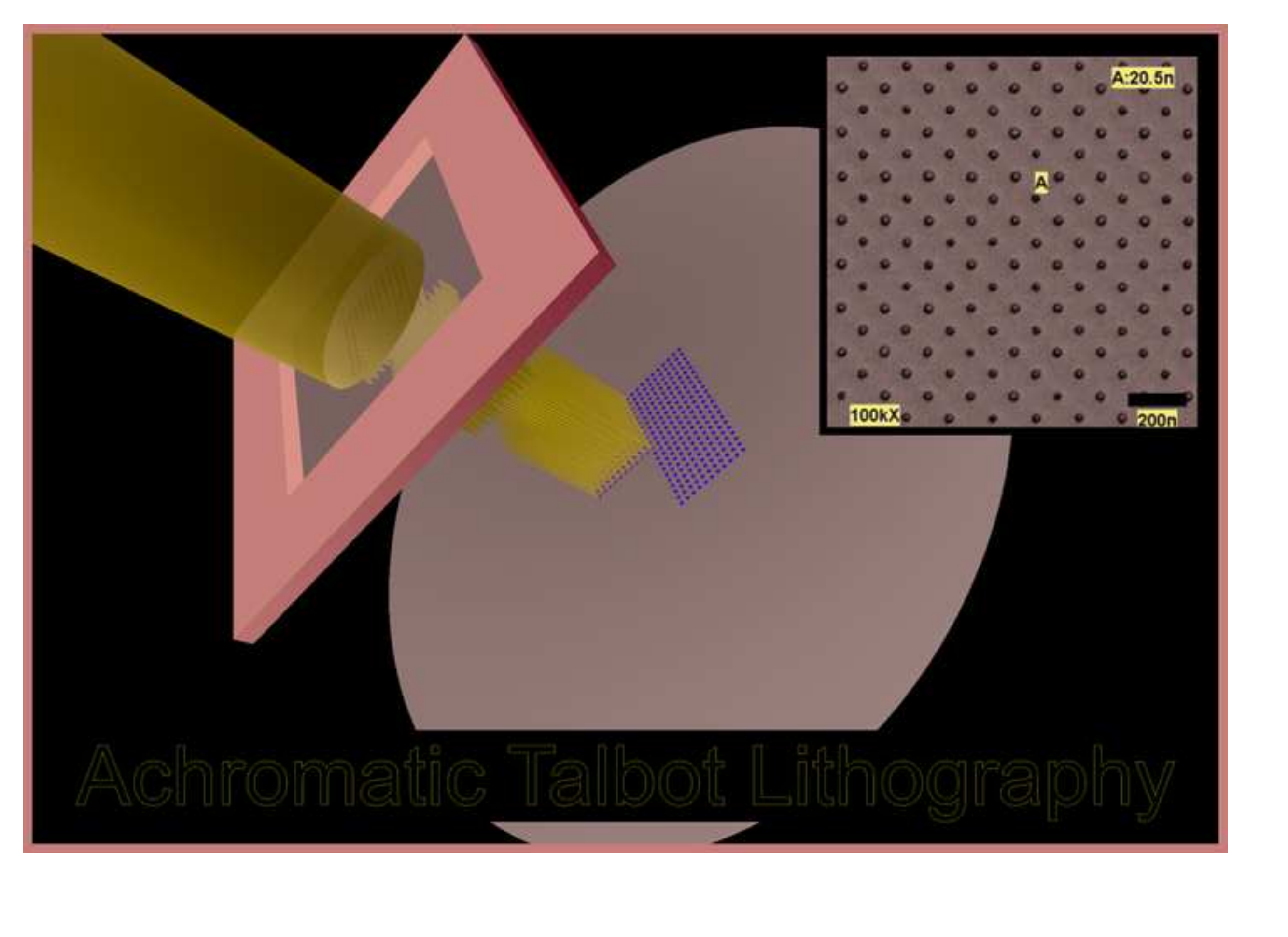

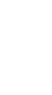
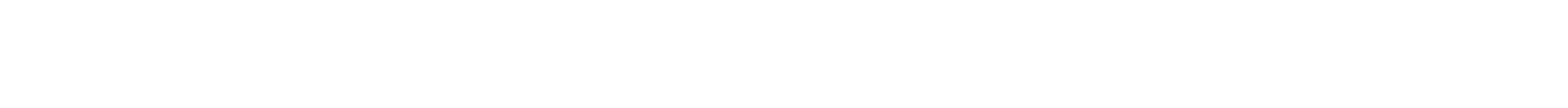